\title{
Estrategias de Negociación y Gestión de Conflictos como Herramienta para los Gerentes
}

\author{
Negotiation Strateǵies and \\ Conflict Management as Tools for Managers
}

Teresa Jesús Ríos Delgado* triosd@unmsm.edu.pe

[RECEPCIÓN: AGOSTO 2016 / CONFORMIDAD: OCTUBRE 2016]

\begin{abstract}
RESUMEN
Este estudio presenta el tema de los conflictos como parte de la interacción humana en las organizaciones por intereses de diversa índole o diferencia de opiniones, siendo el propósito de la administración crear condiciones en las cuales el conflicto como parte de la vida organizacional se controle o se dirija a canales útiles o productivos. La negociación y la gestión de conflictos son herramientas muy importantes de todo gerente en los diversos niveles de la organización, que debe desarrollar a fin de fortalecer la dirección de las organizaciones. La ausencia de estas dos capacidades origina conflictos internos en la organización e impacta en la rentabilidad, conflictos con la comunidad por la contaminación ambiental y, finalmente, con el gobierno local. El estudio se desarrolló basado en la situación de las mypes del sector gráfico que se encuentran en el centro histórico de Lima.
\end{abstract}

Palabras clave: Estrategias de negociación, gestión de conflictos, negociación efectiva

\section{ABSTRACT}

This study presents the topic of conflicts as part of human interaction in organizations by diverse interests or differences of opinion, being the purpose of the administration to create conditions in which the conflict as part of organizational life is controlled or it is directed to useful or productive channels. Negotiation and conflict management are very important tools for managers at all levels of the organization and they must develop in order to strengthen the management of organizations. The absence of these two capabilities creates internal conflicts in the organization that impacts on profitability, also it produces conflicts with the community due to environmental pollution and finally with the local government.

This study was developed based on the situation of MSEs in the graphic sector found in the historic center of Lima.

Key words: negotiation strategies, conflict management, effective negotiation

* Doctora en Educación, Doctora en Gestión y Desarrollo. Magíster en Educación con mención en docencia a nivel superior, abogada, traductora e intérprete. Docente asociada e investigadora del Instituto de Investigaciones de la Facultad de Ciencias Administrativas (FCA) - Universidad Nacional Mayor de San Marcos (UNMSM). Docente de pre y posgrado en Investigación. 


\section{INTRODUCCIÓN}

El conflicto es una lucha, por desacuerdo, incompatibilidad aparente, confrontación de intereses, percepciones o actitudes hostiles entre dos o más partes. El conflicto es connatural con la vida misma, está en relación directa con el esfuerzo por vivir. Los conflictos se relacionan con la satisfacción de las necesidades, se encuentra en relación con procesos de estrés y sensaciones de temor y con el desarrollo de la acción que puede llevar o no hacia comportamientos agresivos y violentos (De Sousa, 2009).

Por otro lado, Ury \& Fisher (1991) señalan que "en innumerables ocasiones se requiere negociación; el conflicto de intereses es una industria en crecimiento. Todas las gentes quieren participar en decisiones sobre problemas que las afectan; pero cada vez menos personas están dispuestas a aceptar decisiones dictadas por otras. Tienen diferencias, y utilizan la negociación para manejarlas. En los negocios, en el gobierno o en la familia, la mayoría de las decisiones se toman mediante negociación. Y aun cuando se comparezca ante un juzgado, casi siempre se negocia un acuerdo antes del juicio".

Como antecedentes podemos señalar a Carbajal Herrera (2008), en la tesis de maestro "Las técnicas de negociación como estrategia para disminuir los conflictos organizacionales en una empresa mediana" en Instituto Politécnico Nacional de México, quien presenta las siguientes conclusiones más importantes:

- La mayoría de las personas trata de evitar los conflictos con el personal de mayor nivel jerárquico.

- La mayoría de miembros presenta desmotivación porque el personal de los niveles más altos no valoran el trabajo efectuado y no resuelven los conflictos equitativamente.

Giménez L, N (2010), en la tesis de grado de magíster en Gerencia empresarial: "La negociación como estrategia gerencial para la solución de conflictos organizacionales en las empresas constructoras de viviendas para el estrato B, ubicadas en Barquisimeto, Estado Lara", en la Universidad Centroccidental Lisandro Alvarado, presenta entre las conclusiones más importantes:

- Se detectó que se presentan desacuerdos para el cumplimiento de los objetivos propuestos en la constructora, la mayoría de veces es porque la planificación no se cumple.

- La toma de decisiones debe caer en una sola persona y no en varias.

Las micro y pequeñas empresas - MYPE - actualmente juegan un rol muy importante en la economía del Perú, tomando fuerza en la economía nacional, debido a que en la mayoría de los casos es cada vez más creciente la migración de los campos a las ciudades, lo que origina mayor concentración de la actividad industrial, además de otros factores propios de cada región, como subversión, narcoterrorismo, falta de oportunidades y otras razones generadas en sus localidades de origen.

En algunas organizaciones, nuestros gerentes no están preparados para manejar conflictos de diversa índole, como personales, laborales o sindicales, lo que afecta directamente a la organización en la consecución de sus objetivos y rentabilidad.

La compresión de las tendencias de mercado en el desarrollo del sistema económico mundial en su conjunto, y el efecto que sobre el Perú tienen, exige analizar la realidad del sector productivo $\mathrm{y}$, en especial, de las micro y pequeñas empresas (MYPES), ya que es evidente que estas tendencias en los últimos años han agotado el patrón de desarrollo industrial basado en el cierre y la protección de mercados locales para dar paso a respuestas de apertura de mercados, caracterizados por una abierta competencia externa e interna.

El conflicto es inevitable en toda organización y, por ende, entre estas. Es así que la presente investigación se justifica porque las organizaciones deben controlar las situaciones de conflicto, siendo estas un pilar en la gestión de organizaciones, por lo cual es importante llevar a cabo esta investigación. Los gerentes deben aprovechar estas situaciones de conflicto para implementar procedimientos que surjan como productos de estrategias de negociación y gestión de conflictos, capaces de crear situaciones productivas para la organización, con procedimientos de gran sentido de responsabilidad e involucrando a los interactuantes en resultados que permitirán bienestar equitativo y constructivo.

El presente estudio tuvo como objetivo establecer qué habilidades de negociación y ges- 
tión de conflictos desarrollar en los gerentes de los diversos niveles de una organización, para afianzar los conocimientos relacionados a las estrategias de negociación y manejo de crisis y minimizar los conflictos existentes con la comunidad y los gobiernos locales.

Se consideró al sector gráfico como unidad de análisis, puesto que se encuentra en un crecimiento progresivo y vislumbra un futuro positivo, lo cual podemos confirmar en las iniciativas de formalización de los pequeños empresarios del centro de Lima, donde se acopian gran cantidad de impresores que han buscado alejarse del seudonegocio de la informalidad; algunos de ellos se han reordenado en las antiguas instalaciones de la empresa Diamante en la avenida Argentina.

Así, a medida que el empresariado tome conciencia sobre las verdaderas repercusiones de trabajar de manera ilegal y de lo transcendental de la formalización, la industria gráfica peruana podrá ganarse un lugar prestigioso en el mercado mundial, donde priman las grandes potencias gráficas.

La zona monumental de la capital, que posee una belleza arquitectónica indescriptible, permaneció 'tomada' por imprentas. Lo paradójico del asunto es que los propios empresarios de dicho rubro son conscientes de la necesidad de reubicarse. El problema es que la propia comuna metropolitana no ha apoyado sus iniciativas, las que han quedado en el olvido o han tenido que ser dejadas de lado. A la fecha, más de 900 imprentas fueron retiradas del centro histórico de Lima y reubicadas en otros lugares de la capital, como parte del plan de acción que ejecuta la Municipalidad Metropolitana para recuperar esa zona declarada Patrimonio de la Humanidad.

De ese número, alrededor de 700 propietarios de estos comercios decidieron retirarse voluntariamente del centro histórico e instalarse en los centros comerciales Unicentro, de la Plaza Unión; Unicachi, en la avenida Caquetá; y en las galerías Arica y Guizado Récord, en Breña, espacios gestionados por la comuna capitalina.

Sin embargo, el desarrollo de la investigación tuvo picos de desazón, ya que llevar a cabo la investigación en pleno desalojo de las empresas -sector gráfico-, del damero de Pizarro, significó que en un momento determinado el estudio se detuviese y con mucha tendencia a no realizarse. Es así que se tuvo que cambiar la hipótesis al fe- nómeno in situ: Las estrategias de negociación y gestión de conflictos son herramientas de gestión en la MYPES del sector gráfico.

\section{MÉTODOS}

Esta investigación es empírica porque es un hecho que ocurre en la sociedad, y aplicada porque se va a enfrentar al problema, proponiendo alternativas de solución.

El nivel de la investigación es explicativo, ya que buscamos conocer los factores causales que explican nuestro problema de investigación. El diseño básico de esta investigación es transeccional-correlacional. Es transeccional, porque nuestro objeto de estudio va a ser analizado en un período determinado de tiempo.

Se elaboró una guía de entrevista y una encuesta para la medición de ambas variables, pero, tal como se explicó anteriormente, hubo algunos problemas en su aplicación por el riesgo en la recolecta de data. Sin embargo, se pudo aplicar una encuesta final, cuyos resultados se pueden observar en los siguientes acápites. La muestra fue criterial, llegando a encuestar a 40 imprentas.

\section{RESULTADOS}

Los diferentes modos de enfrentar el conflicto no son buenos ni malos en sí mismos. Su uso puede ser adecuado o inadecuado, dependiendo de las características del conflicto y las condiciones del contexto. Ahora bien, existen tendencias personales y culturales que favorecen, no inhiben, la expresión de un estilo sobre otro. Lo importante es que, al enfrentar un conflicto, el gerente reflexione acerca de sus reacciones en situaciones similares y dedique algo de su tiempo a explorar opciones que podría emplear para solucionarlo. Seguramente descubrirá que hay más de una manera de hacer frente al problema y posiblemente encontrará una solución que se adapte mejor a las exigencias de la negociación.

La municipalidad de Lima a mediados del 2012, declaró a esta zona como área restringida para este tipo de comercio, según ordenanza edil 1608. La mayoría de imprentas emplea químicos y materiales inflamables y se instalan en casonas de quincha, como la Casa Barbieri. Funcionan, además, en su mayoría, sin licencia de funcionamiento, pues la comuna metropolitana ya no les aprueba o renueva más. He aquí el origen del 
conflicto, los miles de empresarios que trabajan en las aglomeradas imprentas de los jirones Rufino Torrico, Callao y Caylloma no apoyan la propuesta de traslado que la comuna limeña ha elaborado para ellos; la opción presentada por la comuna limeña no les favorece económicamente.

Por su parte, la Municipalidad Metropolitana de Lima, a través de la Gerencia de Desarrollo Económico, promueve el programa Gráficos por Lima, que ha sido creado para fortalecer y promover la labor de los empresarios gráficos, responsables con el desarrollo de nuestra ciudad y que se reubicaron fuera del centro histórico de Lima $(\mathrm{CHL})$, en nuevos centros gráficos con mejores condiciones para el crecimiento y competitividad de la industria gráfica de la ciudad de Lima. Luego del desarrollo de una encuesta, se buscó identificar qué conductas adoptan estas empresas ante el surgimiento de un conflicto. De los resultados que arrojó el estudio, sabemos que el primer paso es siempre recurrir a la parte involucrada en el conflicto para llegar a un acuerdo, sin embargo, la etapa de negociación, en la que las partes se dedican a hacer numerosas llamadas telefónicas, visitas a la contraparte, ofrecimientos, resultaba larga y muchas veces infructuosa. Lo que conlleva una capacidad de respuesta en el buen gobierno que requiere que las instituciones y los procesos traten de servir a todas las partes interesadas en un plazo razonable. También requiere que los responsables de tomar las decisiones sean lo suficientemente flexibles para responder a nuevas situaciones y a las necesidades de los actores sociales (Centro Internacional y Formación. Organizacion Internacional del Trabajo, 2013). Se ha comprobado que los actores involucrados en este conflicto no han podido solucionar el problema planteado, lo que ha generado incertidumbre y pérdidas económicas para ambas partes.

\section{DISCUSIÓN}

Este trabajo presenta un estudio sobre el problema entre la Municipalidad de Lima y las MYPES del sector gráfico, asentadas en el centro histórico de Lima, desde hace 20 años, siendo un sector bastante dinámico, que fue creciendo rápidamente con la aplicación de nuevas tecnologías de impresión y asociando a este como un incipiente clúster que aglomeraba la industria gráfica, los distintos proveedores de materia prima como papel, tinta, así como los proveedores, venta y mantenimiento de toda la gama de equipamiento para este fin. Dada la fuerte demanda de los productos del sector gráfico, generaron un polo de desarrollo en esta zona que está considerada como el centro histórico de Lima, con un gran potencial turístico. La Municipalidad de Lima Metropolitana ha visto este gran crecimiento como una amenaza al ornato y, particularmente, a la seguridad porque en reiteradas oportunidades se han producido incendios en las referidas imprentas por la falta de conciencia de seguridad, agravados por el material de construcción de estas casonas, que básicamente son de adobe y quincha, altamente inflamables, y las grandes cantidades de basura superan aproximadamente las 20 toneladas, que compromete la salud de los trabajadores, quienes laboran en condiciones precarias con insumos químicos altamente contaminantes que afectan la salud pública.

Ambos actores de este conflicto han realizado sus propuestas, las cuales analizamos en la exposición de la presente investigación, y podemos aseverar que en ambas posiciones existe una propuesta de negociación, que afecta claramente el desarrollo de este proceso, aplicando la estrategia de ganar-ganar, que permitiría la sensibilización de ambos actores, poniendo también importancia al tercer actor que ambos no consideran, que es la población, la cual es directamente afectada.

La interpretación de la encuesta aplicada nos permite denotar lo siguiente:

- Un gran sector de las pymes no confía en los operadores de justicia para resolver sus conflictos, es por ello que recurre a acciones de fuerza.

- Asimismo, este sector administra sus conflictos esperando el último momento, pese a que saben de la iniciación del mismo; por otro lado, la única forma de solución de conflictos es el regateo.

- También saben que estos conflictos les ocasionan pérdidas en dinero, en clientes y tiempo, que les va restando competitividad en su sector.

- Queda demostrado que sus conocimientos en las estrategias de negociación y gestión de conflictos es muy precaria, limitándose a utilizar el regateo; otras veces se trata solamente de ganar y el uso de la fuerza. 
Por ello, se recomienda que ambos actores, particularmente las MYPES del sector gráfico, capaciten a sus empresarios en el conocimiento de las estrategias de negociación, como herramienta de gestión en este mundo cada vez más globalizado y competitivo, que genera cada día una serie de conflictos de índole económico-social, y que afectan seriamente la competitividad de dichas empresas.

Estos conocimientos de las estrategias de negociación fortalecerán su competitividad en todo aspecto, porque evitarán las pérdidas de dinero, confianza de los clientes y tiempo.

\section{CONCLUSIONES}

1. En la ocurrencia de este problema está la falta de planificación y de políticas de Estado por parte de la Municipalidad Metropolitana de Lima, desde las anteriores gestiones ediles, pues este problema se fue agudizando desde hace aproximadamente 20 años. Por otro lado, de la presente gestión se puede inferir que no se ha conformado el equipo básico de negociación que permita que este proceso se lleve aplicando el proceso de negociación de ganar - ganar.

2. Las MYPES del sector gráfico de Lima, en el presente proceso de negociación, no han exhibido ninguna capacidad de negociación y solo ha mantenido su posición, sin demostrar capacidad de manejar una estrategia que le permita sacar una ventaja de negociación, y lo único que han conseguido es fragmentarse, ya que un sector importante se ha acogido a los beneficios que propuso la Municipalidad Metropolitana de Lima.

\section{REFERENCIAS BIBLIOGRÁFICAS}

Anzola, M. O. (2003). Una mirada a la cultura corporativa. Editorial Universidad externado de Colombia. Colombia.

Barron, R. (2011). Sistema de financiamiento de las pymes en Lima Metropolitana sector industria gráfica. Ciencias Contables UNMSM, 56.

Berrios, M. (2008). Negociación, mediación y solución de conflictos. Debates Vol XIII, Número 3.Instituto de Estudios Superiores de Admnistración, 35-39.
Cabrera, A. \& De la Cuadra, S. (2009). La pymes, quiénes son, cómo son y qué hacer con ellas. Santiago de Chile: Estudios Públicos.

Camison, Z. (2000). Reflexión sobre la investigación científica de las pymes. Investigación europea de dirección y economías de la empresa Vol $6 \mathrm{~N}^{\circ}$ 2, 13-30.

Centro Internacional y formación. Organización Internacional del Trabajo. (2013). Sistemas de resolución de conflictos laborales. Turín: OIT.

Chiavenato, I. (2014). Introducción a la Teoría General de la Administración. Octava Edición. México. Editorial Mc Graw - Hill

Contreras, C. (28 de abril de 2013). Imprentas saldrían del Centro Histórico de Lima. Imprentas saldrían del Centro Histórico de Lima, pág. 1.

De Sousa, L. (2009). Una mirada genérica a los conflictos. Revista Contribuciones sociales, 12.

Dessler, G. (1979). Organización y Administración. Editorial Prentice-Hall.

Esparza, S. (2005) Programa de desarrollo de personal basado en el diagnóstico de clima organizacional en la empresa Siemens. Tesis para obtener el grado de Lic. en Relaciones Industriales. Universidad de Guanajuato.

Fernandez, L. (18 de enero de 2013). Empresarios gráficos del Centro Histórico no aceptan propuesta de traslado. pág. 1.

Fisher, R., William, U., \& Patton, B. (1999). Si de acuerdo, cómo negociar sin ceder. Bogotá: Norma.

Hackman, J; Oldham, G. (2003). Comportamiento organizacional: conceptos, problemas y prácticas. Editorial McGraw Hill. México DF.

Hellriegel, D. (1998). Administración. Séptima edición. Editorial Soluciones Empresariales. México.

Hernández, E. (2008). Interpretación y análisis de los procesos de negociación. Reflexiones 87 . Universidad de Costa Rica, 87-98.

Herrero, A., \& Puente, J. (2008). Costos de los conflictos empresariales. Debates. Instituto de estudios superiores de admnistración. Vol XIII, Numero 2, 68-72. 
Koontz, H; Welhrich, H (2004). Administración: una perspectiva global. $12^{\circ}$ edición. Editorial McGraw Hill. México.

Ministerio de la Producción (2015). Estadísticas de la dirección general de MYPE y cooperativas.

Municipalidad de Lima Metropolitana (10 de enero de 2013). Programas Gráficos de Lima, empresarios comprometidos con nuestra ciudad. Obtenido de http://www.munlima. gob.pe/programas/ciudad/graficos-porlima\#cuarta-fase

Munch, L. (2005). Métodos y técnicas de investigación. 2da edición. Editorial Trillas. México.
Reyes Ponce, A. (2013). Administración moderna. Editorial Limusa. México.

Robbins, S; Coulter, M. (2010). Administración. $10^{\circ}$ ed. México. Pearson.

Schermerhorn, J. (2010) Adminsitración. Editorial Limusa. México.

Ury, W., \& Fisher, R. (1991). Si de acuerdo cómo negociar sin ceder. Estados Unidos: Norma.

Vega, D. (2011). La negociación como MARC: Aproximaciones al método de Harvard. Revista de Economía y Derecho. Vol 8 , Número 32 UPC, 39-52. 